\title{
Repeat Western Blot Testing After Receiving an HIV Diagnosis and Its Association with Engagement in Care
}

\author{
Wayne A. Duffus ${ }^{*}, 1,2$, Kristina W. Kintziger ${ }^{3}$, James D. Heffelfinger ${ }^{4}$, Kevin P. Delaney ${ }^{4}$, \\ Terri Stephens ${ }^{1}$ and James J. Gibson ${ }^{1}$
}

\author{
${ }^{I}$ South Carolina Department of Health and Environmental Control, Bureau of Disease Control, 1751 Calhoun Street, \\ Columbia, SC 29201, USA \\ ${ }^{2}$ University of South Carolina School Of Medicine, Department of Medicine, Division of Infectious Diseases, Two \\ Medical Park, Columbia, SC 29203, USA \\ ${ }^{3}$ College of Graduate Studies, Georgia Health Sciences University, $112015^{\text {th }}$ Street, Augusta, GA 30912, USA \\ ${ }^{4}$ Division of HIVIAIDS Prevention, Centers for Disease Control and Prevention, 1600 Clifton Road NE, Atlanta, GA \\ 30333, USA
}

\begin{abstract}
Objectives: To examine the prevalence of and factors associated with potentially unnecessary repeat confirmatory testing after initial HIV diagnosis and the relationship of repeat testing to medical care engagement.

Design: South Carolina HIV/AIDS surveillance data for 12,504 individuals who were newly diagnosed with HIV infection between January 1997 and December 2008 were used for this analysis. State law requires that all positive Western blot [WB] results be reported regardless of frequency.

Methods: HIV-infected persons, diagnosed from 1997-2008 and followed through 2009, with repeat positive WB results were compared to those who did not have repeat positive WB results. We defined repeat positive testing as documentation of one or more positive WB obtained $\geq 90$ days following initial WB confirmatory result. HIV care engagement for the period from 2007-2009 was assessed by documentation of CD4+ T-cell/viral load reports to the South Carolina HIV/AIDS surveillance system during each six-month period of a calendar year for those individuals diagnosed prior to the assessment period and still alive at the end. Relative risk [RR] with 95\% confidence intervals [CI] and multivariable general linear models were used to assess if any covariates of interest were independently associated with repeat positive confirmatory testing.

Results: A total of 4,237 [34\%] of 12,504 HIV-infected individuals had results of repeat positive WB testing reported to the surveillance system during 1997-2008. Persons who had repeat positive WB testing were more likely than persons who did not have repeat WB testing to have progressed to AIDS $>1$ year following diagnosis [RR: $1.70 ; 95 \%$ CI: 1.61 , $1.80]$ and to be consistently in care [RR: $1.35 ; 95 \% \mathrm{CI}: 1.24,1.47]$ or have sporadic care [RR: $1.80 ; 95 \% \mathrm{CI}: 1.68,1.94]$.

Discussion: Having repeat positive WB tests may be a marker of engaging HIV care. However, given the limited resources available for care, it is important that healthcare reform policy and clinical recommendations promote improvements in communications about previous test results.
\end{abstract}

Keywords: HIV testing algorithm, repeat positive testing, care engagement, duplicate tests, healthcare reform.

\section{INTRODUCTION}

The primary purpose of HIV testing is to diagnose previously unidentified HIV-infected individuals. Currently in the United States, the algorithm used to diagnose HIV infection includes an Enzyme Linked Immunosorbent Assay [ELISA] followed by confirmatory Western blot [WB] testing. Although a positive HIV serology does not serorevert to negative, HIV-infected persons often undergo additional confirmatory testing following their initial diagnosis. Likely reasons for repeat testing following a

*Address correspondence to this author at the South Carolina Department of Health and Environmental Control, Bureau of Disease Control, HIV/STD Division, 1751 Calhoun Street, Columbia, SC 29201, USA; Tel: (803) 898 0691; Fax: (803) 898 - 2124; E-mail: duffuswa@dhec.sc.gov positive WB test result include as a requirement for enrollment in Ryan White programs or inaccessibility of previous laboratory results when an individual presents for medical care. Because of limited resources additional confirmatory testing after the first positive WB should be kept to a minimum to reduce unnecessary costs.

In 2006, the Centers for Disease Control and Prevention [CDC] published revised recommendations for routine HIV testing in health-care settings [1]. An unintended consequence of expanded screening will likely be an increase in the amount of repeat HIV WB testing when newly diagnosed individuals are referred for medical care from sites that do not typically provide specialty care, such as emergency departments or community-based organizations. For example, following implementation of 
expanded screening in New York City, approximately 63\% of persons testing positive by WB between 2004 and 2006 had previously been diagnosed with HIV infection [2]. This repeat WB testing was therefore not necessary and understanding the full reasons why this occurs could help reduce its frequency and the associated costs.

There is no literature discussing the role of repeat positive confirmatory HIV testing and its relationship to patient care. However, the rationale for repeating a serologic test that does not revert to seronegative over time must be measured by its effect on linkage to and retention in HIV care. The objectives of this investigation were [A] to quantify the frequency of repeat positive confirmatory testing, and [B] to describe associated factors, including those related to healthcare utilization.

\section{METHODS}

\section{Data Source}

In South Carolina [SC], it is mandated that all confirmatory HIV WB test results be reported to the SC Department of Health and Environmental Control [DHEC]. SC DHEC in turn includes all such reports in the enhanced HIV/AIDS Reporting System [eHARS], [3] the national electronic HIV reporting system in the U.S. The SC eHARS is a confidential name-based reporting database maintained by SC DHEC. Reporting newly diagnosed individuals to eHARS is initiated by a variety of entities, including local health departments, correctional facilities, physicians and laboratories. SC eHARS maintains a laboratory record for all new and previous positive confirmatory test results for HIVinfected persons captured by the system. Data quality exceeds the CDC minimum standards on timeliness of reporting $[95 \%$ of cases reported within six months of diagnosis] and completeness of data [ $98 \%$ of cases reported, based on a comparison with other data sources] [SC DHEC unpublished data, 2008]. In addition, SC eHARS is linked with the social security master death files so that deceased HIV-infected individuals are accurately identified. This study was granted an exemption from the SC DHEC Institutional Review Board.

SC eHARS data for 14,013 individuals who were newly diagnosed with HIV infection between January 1997 and December 2008 were used for this analysis. An individual was diagnosed with new HIV infection if they had both an initial positive ELISA and an associated confirmatory WB test result. eHARS variables included in this analysis were date of HIV diagnosis, date of AIDS diagnosis, dates of all positive WB test results, gender, race/ethnicity, age at HIV diagnosis, HIV risk category, date of death [if applicable], county of residence at HIV diagnosis, rural/urban residence at HIV diagnosis, all reported CD4+ T-cell counts and viral loads, and facility source of report of HIV diagnosis. The resulting eHARS data file had 12,504 eligible individuals following removal of duplicate records, records with missing data for HIV or AIDS diagnosis dates, records of individuals with positive HIV test results such as enzyme immunoassay [EIA] or ELISA that did not include an associated positive WB result, and records of persons $<18$ years old or who were not $\mathrm{SC}$ residents at the time of HIV diagnosis.

\section{Outcome Variables}

All positive HIV WB results, irrespective of the number performed per individual, are reported electronically to eHARS and a single positive report is used in this analysis as confirmation of HIV serostatus. The number of separate positive WB results reported was used to define the frequency of repeat HIV confirmatory testing. Positive WB results obtained $<90$ days following the date of an individual's first positive HIV test result were not considered to be repeat tests because the recommendations call for linkage to care within 90 days [1] and to account for hospitalizations during this period [repeat HIV testing may be more likely to occur during hospitalizations soon after diagnosis because initial testing results might not be available], or an individual's decision to reconfirm an initial positive test result soon after diagnosis. Each repeat positive WB test conducted $\geq 90$ days following the initial positive test was considered to be a separate test result [repeat confirmatory test] because linkage to care during this period is not consistent with recommendations. The difference between date of first positive WB result and dates of each subsequent positive WB result conducted $\geq 90$ days later were calculated to determine intervals between repeat testing.

\section{Predictor Variables}

Age at HIV diagnosis was categorized as $18-19$ years, 20-24 years, 25-29 years, 30-39 years, $40-49$ years, and $\geq 50$ years old. Race/ethnicity was categorized as non-Hispanic white, non-Hispanic black, Hispanic/Latino[a], and other [includes Asian, Native Hawaiian/Pacific Islander, Asian/Pacific Islander, American Indian/Alaska Native, and multiracial individuals]. Possible HIV risk category was classified as follows: male-to-male sexual contact, intravenous drug use [IDU], male-to-male sexual contact and IDU, heterosexual contact only [defined by the CDC as someone who has had heterosexual contact with a person known to have HIV or a person at high risk for HIV][4], other risk [including transfusion and transplant, and no identified risk/no risk reported [NIR/NRR]].

County of residence reported at the time of the first positive HIV test result was defined as rural or urban based on the definitions of the SC State Budget and Control Board. A county was considered to be urban if its largest city had at least 25,000 inhabitants, and rural otherwise. Based on this definition, there are 15 urban counties and 31 rural counties in SC [5]. Source of report of the first positive HIV test was categorized as follows: county health department, group practice/private medical doctor, hospital, state facilities [prison and Department of Mental Health], federal facility, out-of-state facility, other [blood bank, other laboratory], and unknown source.

Beginning January 1, 2004, SC state law required all CD4+ T-cell counts and HIV viral load values to be reported to SC DHEC and recorded in the eHARS database. Prior to this time, only CD4+ T-cell counts $<200$ cells $/ \mu \mathrm{L}$ were mandatory report and used to stage a person as having AIDS. Throughout the follow-up period, a CD4+ T-cell count $<200$ cells $/ \mu \mathrm{L}$ was used to classify a person as having 
an AIDS diagnosis. The initial CD4+ T-cell count obtained within one year of the date of the first positive HIV test result was used to define disease stage based on timing of AIDS diagnosis. Individuals who had an AIDS diagnosis within one year of their first positive HIV test result were considered to be diagnosed late in the course of infection [late diagnosis]. Individuals who were not diagnosed with AIDS during follow-up were considered to have been diagnosed early during the course of infection [early diagnosis]. Individuals who received a diagnosis of AIDS greater than one year after diagnosis with HIV were considered to be diagnosed at an intermediate time during the course of their infection [intermediate diagnosis].

The level of engagement in HIV care was assessed by documentation of either a CD4+ T-cell count or HIV viral load report to eHARS in each six-month period of calendar years 2007-2009. Because CD4+ T-cell counts and viral load testing are necessary to make clinical decisions, reporting of these laboratory biomarker values to eHARS in a particular period suggests engagement in HIV medical care during that period [6]. Analysis of care engagement was limited to CD4+ T-cell counts and viral load values reported during 2007-2009 for individuals who were diagnosed with HIV infection from January 1997 to December 2006 and were alive at the end of the study period. Of the 12,504 eligible individuals, 8,677 were diagnosed in 2006 or earlier and were included in the assessment of engagement in HIV care. Persons for whom a CD4+ T-cell count or viral load test was reported in eHARS at least once during each of the 6-month periods between 2007 and 2009 were categorized as being consistently in care. Individuals who did not have any eHARS record of either a CD4+ T-cell count or viral load test during 2007-2009 were categorized as not being in care, and those who had at least one CD4+ T-cell count or viral load test record in eHARS during 2007-2009 but who did not have one of these tests reported during each 6-month period in this interval were classified as sporadic care [7].

\section{Important Study Dates}

For the purposes of our study design, we included individuals diagnosed with HIV in SC from January 1997 through December 2008. All individuals were followed through December 2009. Exclusion of individuals diagnosed prior to 1997 was done for two reasons: 1] to include only individuals diagnosed with HIV post-introduction of HAART, and 2] to limit possible inconsistencies with data entry or collection that might occur over long periods, while still including a substantial sub-section of our cohort. Only individuals diagnosed through December 2008 were included to allow for at least one complete year of follow-up post-HIV diagnosis for proper categorization of individuals as having a late diagnosis or not [see above].

For all analyses of care engagement, we examined the time period between January 2007 and December 2009. CD4 and viral load testing became mandatory in 2004 and we allowed an additional time lag to ensure that the majority of laboratories were in compliance with this law. Therefore, an individual had to be diagnosed with HIV prior to 2007 and still alive at the end of 2009 to be appropriately categorized on level of care engagement. Accordingly, we only included individuals diagnosed with HIV through December 2006 and alive at the end of 2009 .

\section{Statistical Analysis}

For descriptive purposes, frequencies were computed for categorical variables, and univariable associations were determined for individuals who had repeat confirmatory tests compared with those individuals who had no repeat confirmatory test. For non-normally distributed continuous variables, medians and ranges are reported and KruskalWallis tests were used to compare median values. The range from the first to the third quartile is reported for age because of outliers.

Univariable general linear models were used to determine if there was an association between repeat testing and other factors. Multivariable general linear models were used to assess if any covariates of interest were independently associated with repeat positive confirmatory testing. Relative risks [RR] were estimated using a modified Poisson regression method with robust error variance [8]. Adjusted results were obtained by including all statistically significant explanatory variables $[\mathrm{p}<=0.05]$ from univariate models and then using backward elimination to determine the final model by removing the least significant variables until only significant variables remained. RR and 95\% confidence intervals $[\mathrm{CI}]$ are reported. Individuals were excluded from analyses if they had any missing information for age, race/ethnicity, or HIV risk category. Statistical analyses were performed using SAS version 9.1.3 [SAS Institute: Cary, $\mathrm{NC}]$.

\section{RESULTS}

Between January 1, 1997, and December 31, 2008, there were 12,504 study-eligible HIV-infected SC residents reported to eHARS of $20 \%$ progressed to AIDS $>1$ year following HIV diagnosis (Table 1). The overall median age at diagnosis was 36 years, and most diagnoses were among males. The greatest proportion of new diagnoses occurred among non-Hispanic blacks. The most common reported HIV risk categories were heterosexual contact and malemale sex

There were 7,071 [57\%] individuals with at least one additional positive WB reported at some date after initial diagnosis. After classifying all confirmatory tests based on time since initial HIV diagnosis, 8,193/12,504 [65.5\%] persons did not have an additional positive WB $\geq 90$ days after diagnosis and 4,311 [34.5\%] had repeat confirmatory tests $\geq 90$ days following their first positive HIV test date. eHARS captured 7,570 separate positive WB test results among the individuals who had repeat confirmatory testing following initial diagnosis, resulting in a median of 3 [range: 2-14] positive WB results for these persons. The median interval between first positive $\mathrm{WB}$ and any additional confirmatory test following diagnosis was 913 [range: 91 4,658] days. Most of the repeat testing [55\%] occurred within three years of an initial HIV diagnosis. Individuals diagnosed from 2000 through 2008 had the majority of their repeat WB tests occurring within three years, but individuals diagnosed prior to 2000 had the majority occurring greater than three years after diagnosis. Individuals diagnosed with 
Table 1. Overall Demographic Characteristics, Risk Behaviors, and Disease Outcomes Among HIV-Positive Individuals $(\mathbf{N}=12,504)$ - South Carolina, 19972008

\begin{tabular}{|c|c|c|}
\hline Characteristics, Categorical Variables & $\mathbf{N}$ & $\%$ \\
\hline \multicolumn{3}{|l|}{ Positive WB Testing Status ${ }^{l}$} \\
\hline Repeat positive WB results & 8,193 & 65.5 \\
\hline No repeat positive WB results & 4,311 & 34.5 \\
\hline \multicolumn{3}{|l|}{ Gender } \\
\hline Male & 8,641 & 69.1 \\
\hline Female & 3,863 & 30.9 \\
\hline \multicolumn{3}{|l|}{ Age at HIV Diagnosis (years) } \\
\hline $18-19$ & 345 & 2.8 \\
\hline $20-24$ & 1,389 & 11.1 \\
\hline $25-29$ & 1,684 & 13.5 \\
\hline 30-39 & 4,095 & 32.8 \\
\hline $40-49$ & 3,319 & 26.5 \\
\hline $50+$ & 1,672 & 13.4 \\
\hline \multicolumn{3}{|l|}{ Race/Ethnicity } \\
\hline Non-Hispanic, white & 2,879 & 23.0 \\
\hline Non-Hispanic, black & 9,045 & 72.3 \\
\hline Hispanic & 455 & 3.6 \\
\hline Other $^{2}$ & 125 & 1.0 \\
\hline \multicolumn{3}{|l|}{ Residence $^{3}$} \\
\hline Urban & 7,296 & 58.4 \\
\hline Rural & 3,043 & 24.3 \\
\hline Unknown & 2,165 & 17.3 \\
\hline \multicolumn{3}{|l|}{ Risk Category ${ }^{4}$} \\
\hline Male-male sex & 3,843 & 30.7 \\
\hline Heterosexual & 3,831 & 30.6 \\
\hline IDU & 1,033 & 8.3 \\
\hline Male-male sex/IDU & 269 & 2.2 \\
\hline NIR/NRR & 3,502 & 28.0 \\
\hline \multicolumn{3}{|l|}{ Vital Status at Data Linkage } \\
\hline Alive & 10,149 & 81.2 \\
\hline Deceased & 2,355 & 18.8 \\
\hline \multicolumn{3}{|c|}{ Disease Stage Based on Timing of AIDS Diagnosis ${ }^{5}$} \\
\hline Early & 4,976 & 39.8 \\
\hline Intermediate & 2,513 & 20.1 \\
\hline Late & 5,015 & 40.1 \\
\hline \multicolumn{3}{|l|}{ Source of Report $t^{6}$} \\
\hline CHD & 3,224 & 25.8 \\
\hline Group/MD & 2,442 & 19.5 \\
\hline Hospital & 3,475 & 27.8 \\
\hline State facility & 1,051 & 8.4 \\
\hline Federal facility & 394 & 3.2 \\
\hline Another state & 1,367 & 10.9 \\
\hline Other & 468 & 3.7 \\
\hline Unknown & 81 & 0.7 \\
\hline
\end{tabular}

\begin{tabular}{|l|c|c|}
\hline Characteristics, Continuous Variables & Median & Range \\
\hline \hline Age at diagnosis (years) & 36.0 & $(29.0-44.0)^{7}$ \\
\hline Initial CD4+ T-cell count (cells/ $\mu \mathrm{L})$ & 213.0 & $(0.0-2,562.0)$ \\
\hline Initial viral load (copies $/ \mathrm{mL})$ & $23,183.5$ & $(250.0-750,000.0)$ \\
\hline
\end{tabular}

Repeat Positive: eligible study individuals who had additional confirmatory Western blots (WB) tests obtained $>90$ days after initial diagnosis. Each positive WB obtained after $\geq 90$ days was considered a separate result. No Repeat Positive: eligible study individuals who had confirmatory WB tests all obtained $<90$ days after initial diagnosis. All considered a single test for analysis purposes.

${ }^{2}$ Race/Ethnicity: Other includes Asian, Native Hawaiian/Pacific Islander, legacy Asian/Pacific Islander, American Indian/Alaska Native, and multiracial individuals. ${ }^{3}$ Residence is missing for 1,959 observations.

${ }^{4}$ Risk Category: Other modes of exposure, including an adult receiving clotting factor, transfusion, or transplant, were excluded from the analysis due to small numbers $(n=26)$. IDU: intravenous drug use; NIR/NRR: no identified risk/no risk factor reported.

${ }^{5}$ Disease Stage: Intermediate Diagnosis was defined as the development of AIDS $>1$ year after the first positive HIV test; Late Diagnosis was defined as the development of AIDS $<1$ year after the first positive HIV test; and Early Diagnosis was defined as no AIDS diagnosis over the study period.

${ }^{6}$ Source of report (facility type that ordered the testing) of initial HIV diagnosis: CHD = County health department; Group/MD = Group practice/private physician; State facility includes Dept. of Mental Health and state prisons; Other includes blood banks, for insurance purposes, etc.

${ }^{7}$ The range from the $1^{\text {st }}$ to $3^{\text {rd }}$ quartile is reported for age because of outliers.

HIV between 1997 and 2000 accounted for the majority of repeat tests [54\%], indicating that individuals with a longer follow-up period contributed a greater number of repeat WB tests, with the total number of repeat WB tests increasing significantly with earlier years of HIV diagnosis [ $p<0.0001]$. This was further demonstrated by examining year of HIV diagnosis by repeat tester status. For individuals diagnosed between 1997 and 2001, approximately 40\% were repeat testers; between 2002 and 2005, approximately $32 \%$ had any repeat WB test; for 2006 and 2007, 21\% had repeat WB tests; and only $12 \%$ had repeat tests when diagnosed in 2008 . Finally, the median initial CD4+ T-cell count was 240 [range: $0-2,562$ ] cells $/ \mu \mathrm{L}$ for persons with repeat positive WB compared with 196 [range: 0-1,970] cells $/ \mu \mathrm{L}$ for persons without repeat positive WB results $[\mathrm{p}<0.0001]$.

On multivariable analysis, race/ethnicity, risk category, age, disease stage, and source of report were found to be independently associated with repeat confirmatory testing following diagnosis (Table 2). Specifically, non-Hispanic blacks were more likely to have repeat testing compared with non-Hispanic whites. Individuals with all other risk categories were more likely to have repeat testing compared with male-to-male sexual contact. Individuals in all age categories $\geq 25$ years were less likely to have repeat testing compared to individuals aged 18-19 years. Those with an intermediate diagnosis of HIV were more likely to have repeat confirmatory testing compared to those with an early diagnosis. Finally, those who received an initial HIV diagnosis at a group practice/private medical doctor, hospital, or federal facility were less likely to have repeat confirmatory testing, while those diagnosed at a SC state facility, another state, or other type of facility were more likely to have repeat confirmatory testing compared with individuals diagnosed at county health department facilities.

In the subset of 8,677 individuals included in the analysis of engagement in care, the proportion of persons who had either a CD4+ T-cell count or viral load reported to eHARS 
Table 2. Crude and Adjusted Relative Risks between Reported Repeat Positive Western blot (WB) Results and Demographic Characteristics, Risk Behaviors, and Disease Outcomes

\begin{tabular}{|c|c|c|c|c|c|c|c|c|}
\hline Characteristic & \multicolumn{2}{|c|}{ Repeat Positive WB Results ${ }^{1}$} & \multicolumn{2}{|c|}{ No Repeat Positive WB Results ${ }^{1}$} & \multicolumn{2}{|c|}{ Crude } & \multicolumn{2}{|c|}{ Adjusted $^{2}$} \\
\hline Total & 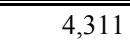 & 34.5 & 8,193 & 65.5 & & & & \\
\hline \multicolumn{9}{|l|}{ Gender } \\
\hline Male & 2,781 & 64.5 & 5,860 & 71.5 & 1.00 & - & & \\
\hline Female & 1,530 & 35.5 & 2,333 & 28.5 & 1.23 & $(1.17,1.29)$ & & \\
\hline $20-24$ & 620 & 14.4 & 769 & 9.4 & 0.89 & $(0.79,1.00)$ & 0.93 & $(0.83,1.04)$ \\
\hline $25-29$ & 645 & 15.0 & 1,039 & 12.7 & 0.76 & $(0.68,0.86)$ & 0.81 & $(0.72,0.91)$ \\
\hline $30-39$ & 1,526 & 35.4 & 2,569 & 31.4 & 0.74 & $(0.66,0.83)$ & 0.78 & $(0.70,0.87)$ \\
\hline $40-49$ & 967 & 22.4 & 2,352 & 28.7 & 0.58 & $(0.52,0.65)$ & 0.62 & $(0.55,0.69)$ \\
\hline $50+$ & 380 & 8.8 & 1,292 & 15.8 & 0.45 & $(0.40,0.52)$ & 0.52 & $(0.45,0.59)$ \\
\hline Hispanic & 125 & 2.9 & 330 & 4.0 & 1.18 & $(1.00,1.39)$ & 1.14 & $(0.97,1.34)$ \\
\hline Other $^{4}$ & 37 & 0.9 & 88 & 1.1 & 1.27 & $(0.96,1.68)$ & 1.15 & $(0.89,1.49)$ \\
\hline \multicolumn{9}{|l|}{ Residence $^{5}$} \\
\hline Urban & 2,494 & 57.9 & 4,802 & 58.6 & 1.00 & - & & \\
\hline Rural & 1,099 & 25.5 & 1,944 & 23.7 & 1.06 & $(0.98,1.13)$ & & \\
\hline Unknown & 718 & 16.7 & 1,447 & 17.7 & 0.97 & $(0.89,1.05)$ & & \\
\hline \multicolumn{9}{|l|}{ Risk Category $^{6}$} \\
\hline Male-male sex & 1,124 & 26.1 & 2,719 & 33.3 & 1.00 & - & 1.00 & - \\
\hline Heterosexual & 1,597 & 37.1 & 2,234 & 27.3 & 1.43 & $(1.34,1.52)$ & 1.36 & $(1.28,1.45)$ \\
\hline IDU & 433 & 10.1 & 600 & 7.3 & 1.43 & $(1.31,1.56)$ & 1.50 & $(1.37,1.63)$ \\
\hline Early & 1,534 & 35.6 & 3,442 & 42.0 & 1.00 & - & 1.00 & - \\
\hline Intermediate & 1,391 & 32.3 & 1,122 & 13.7 & 1.80 & $(1.70,1.90)$ & 1.70 & $(1.61,1.80)$ \\
\hline Late & 1,386 & 32.2 & 3,629 & 44.3 & 0.90 & $(0.84,0.95)$ & 0.99 & $(0.93,1.05)$ \\
\hline \multicolumn{9}{|l|}{ Source of Report $t^{8}$} \\
\hline CHD & 1,256 & 29.1 & 1,968 & 24.0 & 1.00 & - & 1.00 & - \\
\hline Group/MD & 732 & 17.0 & 1,710 & 20.9 & 0.77 & $(0.71,0.83)$ & 0.88 & $(0.82,0.95)$ \\
\hline Hospital & 967 & 22.4 & 2,508 & 30.6 & 0.71 & $(0.67,0.77)$ & 0.85 & $(0.80,0.92)$ \\
\hline State facility & 515 & 12.0 & 536 & 6.5 & 1.26 & $(1.17,1.36)$ & 1.21 & $(1.12,1.30)$ \\
\hline Federal facility & 60 & 1.4 & 334 & 4.0 & 0.39 & $(0.31,0.50)$ & 0.46 & $(0.37,0.59)$ \\
\hline Another state & 536 & 12.4 & 831 & 10.2 & 1.01 & $(0.93,1.09)$ & 1.14 & $(1.05,1.23)$ \\
\hline Other & 224 & 5.2 & 244 & 3.0 & 1.23 & $(1.11,1.36)$ & 1.27 & $(1.15,1.40)$ \\
\hline Unknown & 21 & 0.5 & 60 & 0.7 & 0.67 & $(0.46,0.96)$ & 0.85 & $(0.59,1.24)$ \\
\hline
\end{tabular}

Repeat Positive: eligible study individuals who had additional confirmatory Western blots (WB) tests obtained $\geq 90$ days after initial diagnosis. Each positive WB obtained after $\geq 90$ days was considered a separate result. No Repeat Positive: eligible study individuals who had confirmatory Western blot tests all obtained <90 days after initial diagnosis. All considered a single test for analysis purposes.

${ }^{2}$ Adjusted associations are only reported for the final model. Gender, place of residence, and vital status were not significant in adjusted models and not included in the final model.

${ }^{3} \mathrm{RR}$ indicates relative risk of having a repeat positive Western blot $>90$ days after the initial diagnosis. CI indicates confidence interval.

${ }^{4}$ Race/Ethnicity: Other includes Asian, Native Hawaiian/Pacific Islander, legacy Asian/Pacific Islander, American Indian/Alaska Native, and multiracial individuals.

${ }^{5}$ Residence is missing for 1,959 observations.

${ }^{6}$ Risk Category: Other modes of exposure, including an adult receiving clotting factor, transfusion, or transplant, were excluded from the analysis due to small numbers ( $\mathrm{n}=26$ ). IDU: intravenous drug use; NIR/NRR: no identified risk/no risk factor reported.

${ }^{7}$ Disease Stage: Intermediate Diagnosis was defined as the development of AIDS $>1$ year after the first positive HIV test; Late Diagnosis was defined as the development of AIDS $<1$ year after the first positive HIV test; and Early Diagnosis was defined as no AIDS diagnosis over the study period.

${ }^{8}$ Source of report (facility type that ordered the testing) of initial HIV diagnosis: CHD = County health department; Group/MD = Group practice/private physician; State facility includes Dept. of Mental Health and state prisons; Other includes blood banks, for insurance purposes, etc. 
Table 3. Care Engagement During 2007-2009 for Persons Diagnosed with HIV Infection Between 1997-2006 Categorized by Frequency of Positive HIV Western blot (WB) Test Results

\begin{tabular}{|c|c|c|c|c|c|c|c|c|c|}
\hline \multirow{2}{*}{\multicolumn{2}{|c|}{ Variable }} & \multicolumn{2}{|c|}{ Total $(N=8,677)$} & \multicolumn{2}{|c|}{$\begin{array}{c}\text { Repeat Positive WB } \\
\text { Results }^{1}\end{array}$} & \multicolumn{2}{|c|}{$\begin{array}{c}\text { No Repeat Positive WB } \\
\text { Results }^{1}\end{array}$} & \multirow[t]{2}{*}{ Crude $\mathbf{R R}^{3}$} & \multirow[t]{2}{*}{$95 \% \mathrm{CI}^{3}$} \\
\hline & & $n^{2}$ & $\%$ & $n^{2}$ & $\%$ & $n^{2}$ & $\%$ & & \\
\hline \multicolumn{10}{|c|}{ Period of Care Engagement } \\
\hline 2007 & Jan - Jun & 4,311 & 49.7 & 1,678 & 51.0 & 2,633 & 48.9 & 1.05 & $(1.00,1.11)$ \\
\hline 2008 & Jul - Dec & 4,498 & 51.8 & 1,824 & 55.4 & 2,674 & 49.6 & 1.16 & $(1.09,1.22)$ \\
\hline \multirow{2}{*}{2009} & Jan - Jun & 4,612 & 53.2 & 1,923 & 58.5 & 2,689 & 49.9 & 1.24 & $(1.17,1.31)$ \\
\hline & Jul - Dec & 4,530 & 52.2 & 1,915 & 58.2 & 2,615 & 48.5 & 1.28 & $(1.21,1.35)$ \\
\hline \multicolumn{10}{|c|}{ Level of HIV Care Engagement ${ }^{4}$} \\
\hline \multicolumn{2}{|c|}{ In care } & 2,564 & 29.6 & 921 & 28.0 & 1,643 & 30.5 & 1.35 & $(1.24,1.47)$ \\
\hline
\end{tabular}

${ }^{1}$ Repeat Positive: eligible study individuals who had additional confirmatory Western blots (WB) tests obtained $\geq 90$ days after initial diagnosis. Each positive WB obtained after $\geq 90$ days was considered a separate result. No Repeat Positive: eligible study individuals who had confirmatory WB tests all obtained $<90$ days after initial diagnosis. All considered a single test for analysis purposes.

${ }^{2}$ Number (\%) out of 8,677 study eligible individuals at end of 2006 who had a report to eHARS of either a CD4+ T-cell count or viral load in each 6-month interval from $2007-2009$. ${ }^{3} \mathrm{RR}$ indicates relative risk. CI indicates confidence interval.

${ }^{4}$ "Not in care" defined as having no viral load or CD4 T-cell count reported during 2007-2009; "Sporadic care" defined as having either a viral load or CD4+ T-cell count reported during at least one six-month period but not for all six periods; and "In care" defined as receiving a viral load test or CD4+ T-cell count at least once during each six-month period of 2007, 2008, and 2009.

in the 6-month intervals ranged from $49.7 \%$ to $53.2 \%$ (Table 3). An assessment of the level of care engagement shows that 2,564 [30\%] were consistently in care, 3,491 [40\%] had sporadic care, and 2,622 [30\%] were not in care. Persons who had repeat confirmatory tests after diagnosis were more likely than those who had a single confirmatory test to be consistently engaged in care or have sporadic care during 2007-2009. The frequency of positive WB test results differed by care status: more than one WB test result was reported for $68 \%$ of those individuals in sporadic care, $63 \%$ of those consistently in care, and $54 \%$ of those individuals not in care $[\mathrm{p}<0.0001]$.

\section{DISCUSSION}

More than one-third of individuals included in this analysis had repeat WB confirmatory testing after initial diagnosis. We identified a number of factors that were independently associated with having repeat confirmatory HIV testing, including non-Hispanic black race; reported HIV risk category; younger age; intermediate stage of infection at diagnosis; source of report of initial HIV diagnosis; and sporadic care. However, it is an important surveillance principle that repeat diagnoses are reported each time they occur in order to promote greater completeness for incidence/prevalence of a disease. But HIV serology does not revert to negative and so reducing volume of and need for repeat testing should be a public health goal. Because WB testing is complex, expensive and time-consuming, the occurrence of multiple positive confirmatory WB testing for individuals suggests delayed linkage and poor retention in care and this may impact health outcomes.
For non-Hispanic blacks compared with non-Hispanic whites, possible reasons for repeat confirmatory testing may be that relatively few non-Hispanic blacks had primary care providers, and those who did had low continuity of care with these providers and accessed care from multiple sites as needed $[9,10]$. If medical records are not accessible at the time that care is sought [thus lack of accurate information about testing sites, frequent name and address changes, multiple insurance/medical chart numbers, etc], a WB test may be repeated to confirm diagnosis before HIV medical care is initiated. In addition, African Americans might be more likely than persons from other racial/ethnic groups to get most of their care in urgent care/emergency department settings, [11, 12] and they also may be more likely than others to lack confidence in the initial diagnosis and therefore seek reconfirmation of the diagnosis [13].

Young age is associated with reduced likelihood of returning for test results [14] and being more likely to have unstable living situations [15-18]. These associations will reduce the chances that a young individual will learn the results of HIV testing from providers or public health personnel. Also, younger individuals are less likely than older persons to have health insurance or a primary care provider [19]. Lack of health insurance may result in the use of multiple facilities for healthcare, leading to repeat WB testing when previous HIV test results cannot be easily verified.

There was considerable variability in the association of repeat positive confirmatory testing with facility source of initial HIV diagnosis. Individuals were more likely to have repeat positive WB results if they were initially diagnosed in another state, in a state facility [e.g., prisons] or if the source 
of initial testing was other [e.g., blood bank, insurance purposes], suggesting that one reason for additional testing is to confirm and/or document serostatus as part of engagement or reengagement in care. In SC state facilities [e.g., prisons, Department of Mental Health], individuals are tested on entry and treatment for HIV infection is located within the same facility. Our data do not allow us to determine the pattern of testing on release from prisons or mental health facilities. However, other studies and reports suggest that benefits and continuity of care are frequently not achieved following release, [20-22] and this may lead to increased repeat testing in the community as persons transfer or later re-engage into care, as demonstrated by our data. Better communication between diagnosing and treatment facilities and developing Memorandums of Understanding would serve to reduce delays in confirming infection and initiating care.

Sporadic care may be associated with additional HIV tests being performed when individuals return to medical care or do not disclose their serostatus when HIV testing is offered. Failure to remain in care suggests absence of or inconsistent use of antiretroviral therapy, which might explain why persons who had repeat testing were more likely to progress to AIDS $>1$ year following initial HIV diagnosis. In contrast, progression to AIDS within a year was likely not associated with repeat confirmatory testing because severely immuno-compromised and ill individuals remain engaged in care after linkage and this decreases opportunity for repeat testing. Failure to link and retain persons to care following initial diagnosis is a missed opportunity to allow access to life-saving antiretroviral medications. Use of case management, such as the brief enhanced case management model developed by the Antiretroviral Treatment Access Study [ARTAS], may be one way to link patients quickly and effectively to care after diagnosis, [23, 24] and future studies should identify approaches to increase retention in care [25-30].

Another important reason for repeat testing could be transfer of care to other facilities. Repeat HIV testing is conducted routinely for a new patient during initial patient visits [31]. We found that persons who had evidence of uninterrupted care were more likely to have multiple positive WB results than those not in care and hypothesize that many of these persons had repeat testing upon transfer of care. However, persons who had repeat positive WB results were almost twice as likely to have an intermediate AIDS diagnosis, and it is unlikely that persons diagnosed later in the course of HIV infection derive much benefit from additional HIV testing.

An implication of this analysis for minorities, younger individuals, and those diagnosed later during the course of HIV infection is that having a primary care provider and stability of medical care are key issues related to repeat confirmatory HIV testing and clinical outcomes. Finding ways to promote access to and use of primary care providers and increased stability of care, regardless of the type of insurance a person has, will be important, and healthcare reform may facilitate this.

This analysis has several limitations. First, we used population-level surveillance data which did not include information on knowledge, attitudes and beliefs of individuals. Second, the data do not allow the determination of whether repeat positive WB results were due to initiation of testing by patients or by providers. Because of this, the exact reason[s] for repeat testing cannot be described. Third, surveillance data do not allow determination of the setting in which repeat testing is conducted [e.g., outpatient clinic versus inpatient unit], by the source of report or the type of provider who ordered the testing [e.g., infectious disease specialist, primary care provider, emergency department provider, or other provider]. Fourth, although reporting of WB test results is done by all SC licensed laboratories and is required by law, the completeness of reporting of WB testing by laboratories in SC has not been evaluated. However, reporting bias by laboratories is likely to be minimal as they all report results to the $\mathrm{SC}$ surveillance system electronically. Fifth, it is possible that there was some out-migration of our HIV-infected population that occurred during our follow-up period. Some individuals included in the analysis as not in care or sporadic care may have moved out of state prior to any repeat WB testing or engagement in care. This is a limitation inherent in surveillance data. Sixth, the care status demonstrated from 2007-2009 might not reflect the care status over the period assessed for repeat WB testing. If WB retesting and unstable sources of care were definitively linked, one might expect that the "sporadic care" group relative to the "in care" group would have even greater repeat level of repeat testing than is demonstrated. However, although the timing of the repeat testing relative to the assessment of care status may be separated by many years recent evaluation of care engagement shows that many individuals are not retained after repeat testing.

Finally, there are differential follow-up times for all individuals included in the study. Individuals diagnosed earlier have been followed for more years and have a greater chance of having, and actually do have more, repeat WB tests. We recognize this as a limitation, but we did not wish to exclude any data from this analysis in order for each individual to have the same follow-up period. However, adjustments to the follow-up time were made for certain analyses. We followed all individuals in the study for at least one year post-HIV diagnosis to allow for correct classification of late diagnoses, and we limited our care engagement analysis to only those individuals diagnosed prior to and still alive at the end of the period of interest. Further, varying follow-up times in our study population does not change our conclusions in any way. This data demonstrates that repeat WB tests among known HIVpositive individuals are a problem in our state, regardless of the year of diagnosis and follow-up time.

Our findings suggest that implementing interactive electronic health records in all healthcare systems and finding ways to facilitate sharing of data between systems, while protecting patient confidentiality, might reduce unnecessary repeat testing. One benefit would be to foster timely access to laboratory results by patients and providers, and this may be cost-effective [32] because less time and resources would be used conducting duplicate testing.

The traditional way to confirm [or reconfirm] HIV serostatus with WB testing and many of the likely reasons for repeat testing $>90$ days may not be an effective use of resources because patients are not promptly linked and 
retained in care soon after diagnosis. More emphasis should be placed on finding a simpler testing algorithm and facilitating early linkage and retention in care. Reconfirming serostatus at multiple encounters may satisfy requirements for enrollment and maintenance in Ryan White medication programs, but it may lead to delays in initiation of antiretroviral therapy.

More intensive post-test counseling and enhanced case management that facilitates prompt linkage into HIV care may reduce the amount of repeat positive confirmatory HIV testing that is conducted [23, 24]. However, given expected limitations in resources available for HIV diagnosis and care in the future, it is important that healthcare reform policy and clinical recommendations promote improvements in communications about previous test results that have been conducted and/or the use of easier, faster, and cheaper methods to confirm HIV infection. Easier access to medical records and interactive health information technology could be used to inform providers or prompt them to find previously completed HIV test results. However, unless providers have interactive health information technology, this notification will be limited and many providers will continue to reconfirm past diagnoses.

\section{DISCLAIMER}

The findings and conclusions in this paper are those of the authors and do not necessarily represent the views of the Centers for Disease Control and Prevention.

\section{ACKNOWLEDGEMENT}

Declared none.

\section{CONFLICT OF INTEREST}

The authors confirm that this article content has no conflict of interest.

\section{REFERENCES}

[1] Branson BM, Handsfield HH, Lampe MA, et al. Revised recommendations for HIV testing of adults, adolescents, and pregnant women in health-care settings. MMWR Recomm Rep 2006; 55(RR-14): 1-17.

[2] Hanna DB, Tsoi BW, Begier EM. Most positive HIV western blot tests do not diagnose new cases in New York City: implications for HIV testing programs. J Acquir Immune Defic Syndr 2009; 51(5): 609-14.

[3] CDC. Statistics and Surveillance. Questions and answers: changes to the hiv surveillance report and general surveillance report questions 2010. Available from: http://www.cdc.gov/hiv/topics/ surveillance/resources/qa/generalqa.htm [cited: April 26 2010].

[4] CDC. Technical Notes. HIV Surveillance Report, 2009. Available from: http://www.cdc.gov/hiv/topics/surveillance/resources/reports/ [cited: November 22 2011]

[5] SCBCB. Data about South Carolina and its people, 2010. Available from: http://www.bcb.sc.gov/BCB/BCB-sc-data.phtm [cited: April 26 2010]

[6] DHHS. Panel on Antiretroviral Guidelines for Adults and Adolescents. Guidelines for the use of antiretroviral agents in HIV1-infected adults and adolescents. Department of Health and Human Services 2009

[7] Olatosi BA, Probst JC, Stoskopf CH, Martin AB, Duffus WA. Patterns of engagement in care by HIV-infected adults: South Carolina, 2004-2006. AIDS 2009; 23(6): 725-30.
[8] Zou G. A modified poisson regression approach to prospective studies with binary data. Am J Epidemiol 2004; 159(7): 702-6.

[9] Merzel C, Moon-Howard J. Access to health services in an urban community: does source of care make a difference? J Urban Health 2002; 79(2): 186-99.

[10] Shi L. Experience of primary care by racial and ethnic groups in the United States. Med Care 1999; 37(10): 1068-77.

[11] Hing E, Hall MJ, Xu J. National Hospital Ambulatory Medical Care Survey: 2006 outpatient department summary. Natl Health Stat Rep 2008; 6(4): 1-31.

[12] Hong R, Baumann BM, Boudreaux ED. The emergency department for routine healthcare: race/ethnicity, socioeconomic status, and perceptual factors. J Emerg Med 2007; 32(2): 149-58.

[13] Bohnert AS, Latkin CA. HIV testing and conspiracy beliefs regarding the origins of HIV among African Americans. AIDS Patient Care STDS 2009; 23(9): 759-63.

[14] Simundic AM, Nikolac N, Miler M, Cipak A, Topic E. Efficiency of test report delivery to the requesting physician in an outpatient setting: an observational study. Clin Chem Lab Med 2009; 47(9): 1063-6.

[15] The US Conference of Mayors. A status report on hunger and homelessness in America's cities: A 23-city survey 2007. Available from: http://www.usmayors.org/hhsurvey2007/hhsurvey07.pdf [cited: February 10 2011]

[16] The Second Annual Homeless Assessment Report to Congress US Department of Housing and Urban Development Office of Community Planning and Development, 2008. Available from: http://www.hudhre.info/documents/2ndHomelessAssessmentRepor t.pdf [cited: February 10 2011]

[17] Kushel MB, Yen IH, Gee L, Courtney ME. Homelessness and health care access after emancipation: results from the Midwest Evaluation of Adult Functioning of Former Foster Youth. Arch Pediatr Adolesc Med 2007; 161(10): 986-93.

[18] Rosenthal D, Rotheram-Borus MJ, Batterham P, Mallett S, Rice E, Milburn NG. Housing stability over two years and HIV risk among newly homeless youth. AIDS Behav 2007; 11(6): 831-41.

[19] Callahan ST, Cooper WO. Changes in ambulatory health care use during the transition to young adulthood. J Adolesc Health 2010; 46(5): 407-13.

[20] Baillargeon J, Giordano TP, Rich JD, et al. Accessing antiretroviral therapy following release from prison. JAMA 2009; 301(8): 84857.

[21] Baillargeon JG, Giordano TP, Harzke AJ, Baillargeon G, Rich JD, Paar DP. Enrollment in outpatient care among newly released prison inmates with HIV infection. Public Health Rep 2010; 125(Suppl 1): 64-71.

[22] Springer SA, Pesanti E, Hodges J, Macura T, Doros G, Altice FL. Effectiveness of antiretroviral therapy among HIV-infected prisoners: reincarceration and the lack of sustained benefit after release to the community. Clin Infect Dis 2004; 38(12): 1754-60.

[23] Craw JA, Gardner LI, Marks G, et al. Brief strengths-based case management promotes entry into HIV medical care: results of the antiretroviral treatment access study-II. J Acquir Immune Defic Syndr 2008; 47(5): 597-606.

[24] Gardner LI, Metsch LR, Anderson-Mahoney P, et al. Efficacy of a brief case management intervention to link recently diagnosed HIV-infected persons to care. AIDS 2005; 19(4): 423-31.

[25] Giordano TP, Gifford AL, White AC Jr, et al. Retention in care: a challenge to survival with HIV infection. Clin Infect Dis 2007; 44(11): 1493-9.

[26] Konkle-Parker DJ. How can we facilitate entry into and retention in HIV care? HIV Clin Winter 2010; 22(1): 10-1.

[27] Mugavero MJ. Improving engagement in HIV care: what can we do? Top HIV Med 2008; 16(5): 156-61.

[28] Naar-King S, Bradford J, Coleman S, Green-Jones M, Cabral H, Tobias C. Retention in care of persons newly diagnosed with HIV: outcomes of the Outreach Initiative. AIDS Patient Care STDS 2007; 21(Suppl 1):S40-8.

[29] Naar-King S, Green M, Wright K, Outlaw A, Wang B, Liu H. Ancillary services and retention of youth in HIV care. AIDS Care 2007; 19(2): 248-51. 
[30] Tobias C, Cunningham WE, Cunningham CO, Pounds MB. Making the connection: the importance of engagement and retention in HIV medical care. AIDS Patient Care STDS 2007; 21(Suppl 1): S3-8.

[31] DHHS. Guidelines for the use of antiretroviral agents in HIV-1infected adults and adolescents. 2009 December $1^{\text {st }}$. Available from: http://aidsinfo.nih.gov/Guidelines/GuidelineDetail.aspx?Men uItem $=$ Guidelines $\&$ Search $=$ Off $\&$ GuidelineID $=7 \&$ ClassID $=1 \quad$ [cited: September 30 2010].

[32] Wang SJ, Middleton B, Prosser LA, et al. A cost-benefit analysis of electronic medical records in primary care. Am J Med 2003; 114(5): 397-403.

(C) Duffus et al.; Licensee Bentham Open.

This is an open access article licensed under the terms of the Creative Commons Attribution Non-Commercial License (http://creativecommons.org/licenses/by-nc/ $3.0 /$ ) which permits unrestricted, non-commercial use, distribution and reproduction in any medium, provided the work is properly cited 